\section{Dermoscopic findings of pseudoxanthomatous mastocytosis localized on vulva*}

\section{Qiaofei $\mathrm{Li}^{1}$}

Kang Zeng ${ }^{1}$

Xiaoming Peng 1

Fang Wang 2

\section{DOI: http:/ /dx.doi.org/10.1590/abd1806-4841.20188039}

\section{Dear Editor,}

Mastocytosis is a group of diseases characterized by proliferation and accumulation of mast cells in various organs such as the skin, bone marrow, liver, spleen, and lymph nodes. ${ }^{1}$ Cutaneous mastocytosis $(\mathrm{CM})$ is the most common presentation and can be subclassified into three clinical variants: maculo-papular cutaneous mastocytosis (which is further subdivided into urticaria pigmentosa, telangiectasia macularis eruptiva perstans and papular/plaque variant), diffuse cutaneous mastocytosis, and solitary mastocytoma. ${ }^{1}$ Pseudoxanthomatous mastocytosis is a rare variety of diffuse cutaneous mastocytosis and has been introduced to describe the presence of yellowish papular or nodular lesions. ${ }^{1,2}$ Although the manifestations of $\mathrm{CM}$ are diverse, it has been reported that its dermoscopic pattern is distinguishable. ${ }^{3}$ As far as we know, few reports have described dermoscopic features of pseudoxanthomatous mastocytosis. ${ }^{3-5}$ Herein, we report a case of pseudoxanthomatous mastocytosis occurring on a girl's vulva and profile its dermoscopic findings.

An 8-year-old girl presented with a 6-year history of asymptomatic cutaneous lesions on her vulva. On physical examination, several firm, round to ovoid, skin-colored to yellowish papules and nodules varying in size from $2 \mathrm{~mm}$ to $1 \mathrm{~cm}$ in diameter, with a negative Darier's sign, were seen on the labia majora bilaterally. Some nodular lesions had orange peel-like pits on the surface (Figure 1). Laboratory tests including complete blood count, liver and kidney function, and tryptase level were performed and the results were

Received 28 December 2017.

Accepted 31 March 2018.

* Work conducted at the Department of Dermatology, Nanfang Hospital, Southern Medical University, Guangzhou 510515, China; Department of Dermatology, The First Affiliated Hospital, Sun Yat-sen University, Guangzhou 510080, China.

Financial support: None.

Conflict of interest: None.

1 Department of Dermatology, Nanfang Hospital, Southern Medical University, Guangzhou 510515, China.

2 Department of Dermatology, The First Affiliated Hospital, Sun Yat-sen University, Guangzhou 510080, China.

MAILING ADDRESS:

Fang Wang

E-mail: ffwang.640@163.com; wangf78@mail.sysu.edu.cn

C2018 by Anais Brasileiros de Dermatologia all within normal range. Abdominal ultrasonography did not show hepatosplenomegaly or any other abnormalities.

Dermoscopy examination of a nodular lesion showed pigmented stripes radiated from hair follicles on a pink background. Linear branched and reticular vascular patterns were present as well (Figure 2). Histopathology examination of the skin biopsy revealed hyperpigmentation of keratinocytes in the basal layer and dense infiltration of mast cells with amphophilic granular cytoplasm and round-shaped nucleus in the entire dermis (Figure 3). The infiltrating cells were positively stained for CD117. Based on these findings, the diagnosis of pseudoxanthomatous mastocytosis was established. No treatment was introduced because the patient

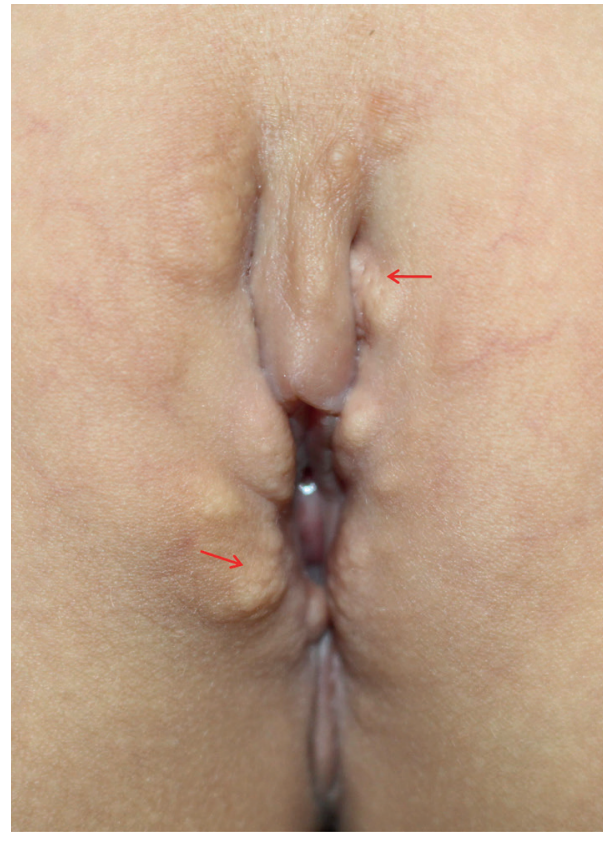

Figure 1:

Several firm round to ovoid skin-colored to yellowish papules and nodules varying in size were seen on the patient's labia majora bilaterally. Orange peellike pits on the surface are shown (indicated by red arrows)

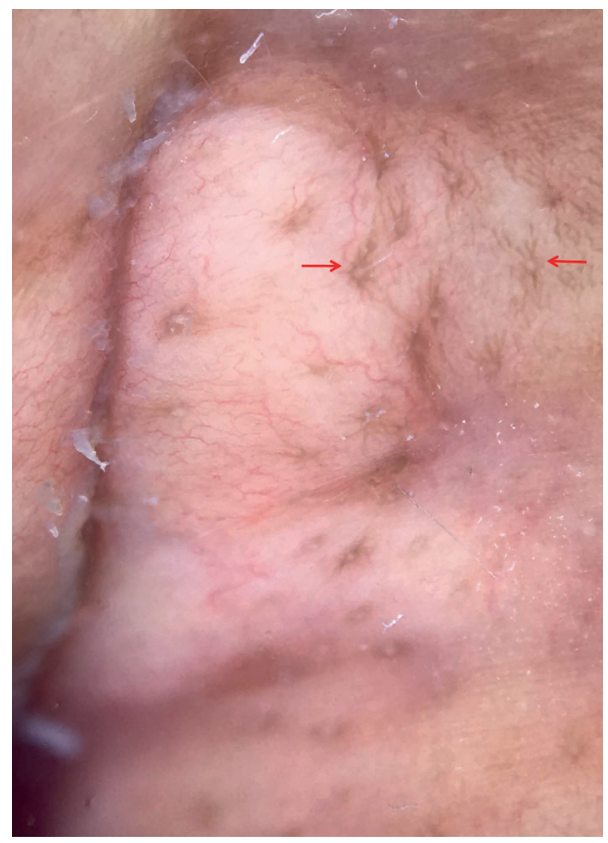

Figure 2:

Dermoscopy examination of a nodular lesion revealed pigmented stripes radiated from hair follicles (indicated by red arrows) on a pink background, as well as linear branched and reticular vascular patterns 
was asymptomatic. The patient had been followed-up for 4 months and is still undergoing follow-up.

Pseudoxanthomatous mastocytosis is extremely rare. According to the clinical data published to date, the terms "xanthelasmoid", "nodular" and "pseudoxanthomatous" mastocytosis describe all the same clinical condition and therefore could be considered as its synonyms. ${ }^{2}$ Pseudoxanthomatous mastocytosis more commonly develops during childhood. Although the exact prevalence of the disease is not clear, based on a previous study, it was observed in 10 of 280 mastocytosis patients. ${ }^{1}$ The lesions can be localized or disseminated, presenting as homogeneous yellow or cream-colored papules and nodules, varying in size, and without the classic Darier's sign. Cerebriform or "peau d'orange" surface is a distinctive feature. No specific predilection site has been reported in this disease. The lesions could gradually spread to the whole body in some generalized cases. ${ }^{1}$ In the present case and in the patient reported by Pérez-Pérez $\mathrm{L}$ et $a l^{2}{ }^{2}$ the lesions occurred on the vulva, thus, we can infer the vulva might be a predilection site in localized cases. Clinical differential diagnoses include pseudoxanthoma elasticum, juvenile xanthogranuloma and xanthoma, among others. The diagnosis is confirmed on the basis of clinical features and characteristic histopathological findings.

To date, four distinguishable dermoscopic structures of mastocytosis have been identified: light-brown blot, pigment network, reticular vascular pattern, and yellow-orange blot. ${ }^{3-5}$ The structures of light-brown blot and pigment network are prevalent in patients with maculo-papular mastocytosis, whereas the pattern of reticular vessels is related to telangiectasia macularis eruptiva perstans and the yellow-orange blot could be a clue for the diagnosis of mastocytoma. ${ }^{3-5}$ In addition, the presence of a reticular vascular pattern might be a sign of increased need for daily antimediator therapy. ${ }^{3}$ From the present case, we believe that the dermoscopic feature constituted by pigmented stripes radiated from hair follicles, could be a specific sign in pseudoxanthomatous mastocytosis. It is believed that the pigment network seen with dermoscopy is due to melanocyte proliferation and melanin pigment production

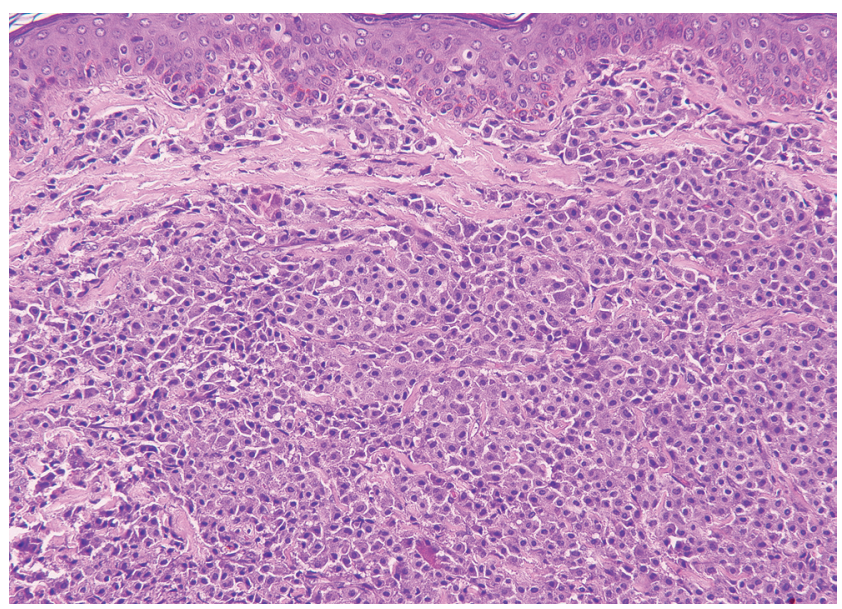

Figure 3: Histopathology of the skin lesion revealed hyperpigmentation of basal layer and diffuse infiltration of mast cells with amphophilic granular cytoplasm and round-shaped nucleus in the entire dermis (Hematoxylin \& eosin, x200) stimulated by a high local concentration of mast cell growth factor (MGF, is also known as Kit-ligand, stem cell growth factor, and steel factor). ${ }^{5}$ The biopsy of our case showed the hyperpigmentation in basal layer was correlated with the pigmented stripes disclosed by dermoscopy.

In summary, we report a rare case of pseudoxanthomatous mastocytosis and describe its specific dermoscopy features, which is a diagnostic clue to such cases. $]$

\section{REFERENCES}

1. Lange M, Niedoszytko M, Nedoszytko B, Łata J, Trzeciak M, Biernat W. Diffuse cutaneous mastocytosis: analysis of 10 cases and a brief review of the literature. J Eur Acad Dermatol Venereol. 2012;26:1565-71.

2. Pérez-Pérez L, Allegue F, Caeiro JL, Fabeiro JM, Pérez Rodríguez A, Zulaica A Coexistence of two types of clinical lesions in childhood-onset mastocytosis. Indian J Dermatol Venereol Leprol. 2011;77:184-7.

3. Vano-Galvan S, Alvarez-Twose I, De las Heras E, Morgado JM, Matito A, SánchezMuñoz L, et al. Dermoscopic features of skin lesions in patients with mastocytosis. Arch Dermatol. 2011;147:932-40.

4. Unterstell N, Lavorato FG, Nery NS, Mann D, Alves Mde F, Barcauí C. Dermatoscopic findings in telangiectasia macularis eruptiva perstans. An Bras Dermatol. 2013;88:643-5.

5. Akay BN, Kittler H, Sanli H, Harmankaya K, Anadolu R. Dermatoscopic findings of cutaneous mastocytosis. Dermatology. 2009;218:226-30.

AUTHORS CONTRIBUTIONS
Qiaofei Li
Approval of the final version of the manuscript, Conception and planning of the study,
Elaboration and writing of the manuscript, Obtaining, analyzing and interpreting the
data, Intellectual participation in propaedeutic and/or therapeutic conduct of the cases
studied, Critical review of the literature, Critical review of the manuscript
Kang Zeng
Approval of the final version of the manuscript, Obtaining, analyzing and interpreting
the data, Critical review of the literature, Critical review of the manuscript
Xiaoming Peng
Approval of the final version of the Obtaining, analyzing and interpreting the data, In-
tellectual participation in propaedeutic and/or therapeutic conduct of the cases studied,
Critical review of the manuscript
Fang Wang
Approval of the final version of the manuscript, Conception and planning of the study,
Elaboration and writing of the manuscript, Obtaining, analyzing and interpreting the
data, Intellectual participation in propaedeutic and/or therapeutic conduct of the cases
studied, Critical review of the literature, Critical review of the manuscript

How to cite this article: Li Q, Zeng K, Peng X, Wang F. Dermoscopic findings of pseudoxanthomatous mastocytosis localized on vulva. An Bras Dermatol. 2018;93(6):940-1. 